\title{
Electronic Portal Imaging
}

National Cancer Institute

\section{Source}

National Cancer Institute. Electronic Portal Imaging. NCI Thesaurus. Code C104994.

The acquisition of images of an anatomic area that is being irradiated using radiotherapy beams and an electronic detector. 